\title{
Smeared branes and the Gubser-Mitra conjecture
}

\author{
Paul Bostock and Simon F. Ros \\ Centre for Particle Theory, Department of Mathematical Sciences \\ University of Durham, South Road, Durham, DH1 3LE, UK
}

(Dated: October 27, 2018)

\begin{abstract}
We show that smeared brane solutions, where a charged black $p$-brane is smeared uniformly over one of the transverse directions, can have a Gregory-Laflamme type dynamical instability in the smeared direction even when the solution is locally thermodynamically stable. These thus provide counterexamples to the Gubser-Mitra conjecture, which links local dynamical and thermodynamic stability. The existence of a dynamical instability is demonstrated by exploiting an ansatz due to Harmark and Obers, which relates charged solutions to neutral ones.
\end{abstract}

\section{INTRODUCTION}

One of the most remarkable features of black holes is the connection between properties of the classical solutions and thermodynamics. The realisation that the laws of black hole mechanics (describing some dynamical aspects of black hole horizons) can be identified with the laws of thermodynamics [1, 2] is at the origin of more than 30 years of work using black hole thermodynamics to gain clues about the form of the quantum theory of gravity.

Black $p$-brane solutions in more than four spacetime dimensions are richer dynamical systems, exhibiting new behaviours that have no analogues for black hole solutions. In particular, some $p$-brane solutions suffer from a classical instability discovered by Gregory and Laflamme [3, 4]. They showed that perturbations of the metric with wavelength longer than some critical wavelength in the extended directions grow exponentially. Thus, the perturbation breaks translational invariance in the extended directions. The existence of this instability raises two fundamental questions:

- Given that a solution is unstable, what is the final state of the instability?

- Can the connection between classical dynamics and thermodynamics be extended to include this feature?

These questions have been the focus of considerable activity. In [3], a heuristic argument for the appearance of the instability was advanced, based on comparing the entropy of the $p$-brane to that of a periodic array of black holes. This represents a first attempt to make a connection between this instability and thermodynamics, and at the same time suggests that the end state for the instability might be such an array of black holes.

More recently, a more precise connection between dynamics and thermodynamics was conjectured by Gubser and Mitra [5], who suggested that a black brane with a non compact translational symmetry is classically stable if and only if it is locally thermodynamically stable ${ }^{1}$. This conjecture was partially motivated by the dual description of such black $p$-branes in string theory in terms of a finite-temperature gauge theory on the $p$-brane's worldvolume. A partial proof of this conjecture was given for a certain class of $p$-branes by Reall $[\underline{6}$. The proof is based on relating the threshold unstable mode, which has wavelength equal to the critical wavelength, to a negative mode of the Euclidean black hole solution. Further investigations of this relation were carried out in [7, 8, 9, 10]; the results so far support the conjecture. Studies of the connection between dynamical and thermodynamic instability which relax the requirement of translational invariance appear in [11, 12, 13].

Our aim is to extend these investigations of the conjecture to study smeared branes: that is, we take a $p$-brane and smear it uniformly over one of the transverse directions, and study stability to perturbations in this smeared direction. This is a natural extension of the investigation of $p$-branes in 6, 8, 9, 10]. The two classes of solutions are related by T-duality, which implies that the thermodynamics of the smeared branes is identical to that of the $p$-brane with the same total number of extended directions. However, the study of perturbations in the smeared direction is technically more challenging; certain simplifications exploited in [ 6 ] no longer apply. We will exploit recent advances in the construction of non-uniform brane solutions to circumvent this problem.

\footnotetext{
*Electronic address: P.B.Bostock@durham.ac.uk

${ }^{\dagger}$ Electronic address: S.F.Ross@durham.ac.uk

1 Thermodynamic stability is taken to mean that the Hessian of the entropy (thought of as a function of extensive variables such as the charge and mass of the solution) has no positive eigenvalues.
} 
This work on non-uniform solutions was inspired by a contemporaneous development concerning the second question above: Horowitz and Maeda [14] argued that the horizon could not pinch off, so the end state of the instability could not be a collection of separate black holes. Instead, they conjectured that the solution would settle down to a nontranslationally invariant solution with the same horizon topology as the original $p$-brane. Examples of solutions which are non-uniform in one extended direction were subsequently found by perturbing the neutral uniform black string by the threshold unstable mode, which generates a branch of static non-uniform solutions [15, 16] (this was recently extended to higher dimensions [17]). These non-uniform black strings have too large a mass to be the endpoint of the instability, but they show that non-uniform solutions with regular event horizons do exist ${ }^{2}$.

In [19], Harmark and Obers presented a useful ansatz for such non-uniform solutions. Their ansatz, which will be reviewed in detail in the next section, describes both vacuum black strings of the type discussed in [15, 16] and charged black branes smeared over a transverse circle. The ansatz involves just two undetermined functions, but in the vacuum case, it has been shown to be equivalent to the general conformal ansatz, which involves three undetermined functions 20,21$]$.

In this paper, we will use some of these results from the study of the possible end states of the instability to show that smeared charged black holes provide a counter-example to the Gubser-Mitra conjecture, implying that the connection between dynamical instability and thermodynamics is more complicated than previously thought. The key is that in the Harmark and Obers ansatz, the full equations of motion are satisfied if the two undetermined functions satisfy a system of equations which are independent of the charge [19]. Hence, any solution in the vacuum case gives a solution in the charged case. In particular, the branch of non-uniform solutions meeting the black string at the threshold unstable mode found in [16] must persist for non-zero charge. That is, there is a threshold unstable mode for any charge. If we increase the charge sufficiently, the uniform solution becomes thermodynamically stable; however, we take the existence of the threshold unstable mode to indicate that it remains dynamically unstable, violating the Gubser-Mitra conjecture ${ }^{3}$.

As in [6], our analysis involves only the study of static solutions, so we do not explicitly find the instability. However, the fact that the 'phase diagram' of static solutions is qualitatively unchanged as the charge varies strongly argues that there is still an instability. Our result certainly obstructs any attempt to extend the argument of [ 6 ] to such smeared charged string solutions.

It is surprising that the addition of a smeared charge, which certainly affects the thermodynamic properties of the black brane solution, does not affect the dynamics of the perturbations described by the Harmark and Obers ansatz. It would be very interesting to understand this observation from a dual field theory point of view. Such an understanding might also help to see how the Gubser-Mitra conjecture can be modified or reformulated in light of these results.

The next section reviews the essential features of the Harmark and Obers ansatz, and uses them to show that there is a threshold unstable mode for a smeared charged black hole. In the appendix, we discuss the extent to which this ansatz provides a general description for charged smeared branes.

\section{HARMARK AND OBERS' ANSATZ}

In [19], an ansatz for charged dilatonic black hole solutions on a cylinder $\mathbb{R}^{d-1} \times S^{1}$ was introduced. This ansatz was motivated by introducing a coordinate system which interpolates between the usual black brane with transverse space $\mathbb{R}^{d}$, which is a good description of a black hole on a cylinder of small mass, and the black brane smeared on the transverse circle, which is a good description at large mass. The ansatz is

$$
\begin{gathered}
d s_{n}^{2}=H^{-\frac{d-2}{n-2}}\left(-f d t^{2}+\sum_{i=1}^{p}\left(d x^{i}\right)^{2}+H R_{T}^{2}\left(f^{-1} A d R^{2}+\frac{A}{K^{d-2}} d v^{2}+K R^{2} d \Omega_{d-2}^{2}\right)\right) \\
e^{a \phi}=H^{2}, \quad A_{01 . . p}=\operatorname{coth} \alpha\left(1-H^{-1}\right), \quad f=1-\frac{R_{0}^{d-3}}{R^{d-3}}, \quad H=1+\frac{R_{0}^{d-3} \sinh ^{2} \alpha}{R^{d-3}},
\end{gathered}
$$

where $A$ and $K$ are two unknown functions of $R$ and $v$ only, and the total spacetime dimension $n=d+p+1$. This solution has an event horizon at $R=R_{0}$.

\footnotetext{
${ }^{2}$ Preliminary numerical investigations of the endpoint of the Gregory-Laflamme instability were reported in [18.

${ }^{3}$ In a recent paper [22], it was suggested that gyrating strings might also give a counterexample to the Gubser-Mitra conjecture.
} 
The uniform smeared black $p$-brane is given by setting $A=K=1$. It's thermodynamics are equivalent to those of the T-dual $p+1$-brane solution. In particular, the mass and charge $\operatorname{are}^{4}$

$$
\begin{gathered}
M=\frac{\Omega_{d-2} 2 \pi R_{T} V_{p}}{16 \pi G}\left(R_{T} R_{0}\right)^{d-3}\left[(d-2)+(d-3) \sinh ^{2} \alpha\right], \\
Q=\frac{\Omega_{d-2} 2 \pi R_{T}}{16 \pi G}\left(R_{T} R_{0}\right)^{d-3}(d-3) \sinh \alpha \cosh \alpha,
\end{gathered}
$$

while the entropy and temperature are

$$
S=\frac{\Omega_{d-2} 2 \pi R_{T} V_{p}}{4 G}\left(R_{T} R_{0}\right)^{d-2} \cosh \alpha, \quad T=\frac{d-3}{4 \pi\left(R_{T} R_{0}\right) \cosh \alpha} .
$$

The statement of the conjecture uses the Hessian matrix of derivatives of the entropy as the test for thermodynamic stability. However, following 6, 8, 9], we will assume that there is no charged field in the theory, so the charge is not allowed to vary, and focus on the specific heat: we take the condition for thermodynamic stability to be the positivity of the specific heat,

$$
C_{Q}=\left(\frac{\partial M}{\partial T}\right)_{Q}>0
$$

It is easy to work out from these formulae that the specific heat is negative at $Q=0$ for all values of $d$, but it becomes positive above some critical charge if $d>5$.

We now review the study of non-uniform solutions in this ansatz. In [19], it was shown that when we impose the equations of motion

$$
\begin{gathered}
R_{\mu \nu}-\frac{1}{2} \partial_{\mu} \phi \partial_{\nu} \phi-\frac{1}{2} e^{a \phi} F_{\lambda \mu} F_{\nu}^{\lambda}=g_{\mu \nu}\left(\frac{1}{4(2-n)}\right) e^{a \phi} F^{2} \\
\nabla^{2} \phi=\frac{a}{4} e^{a \phi} F^{2}, \quad \nabla_{\mu}\left(e^{a \phi} F^{\mu \nu}\right)=0
\end{gathered}
$$

the resulting system of equations for $A$ and $K$ is independent of the charge (i.e., of $\alpha$ ), and hence also of the value of $p$ (since the extra dimensions $x^{i}$ decouple in the neutral case). Furthermore, the boundary condition necessary to ensure regularity at the horizon is simply that $A\left(R_{0}, v\right)$ and $K\left(R_{0}, v\right)$ are constants, so the boundary conditions also do not involve the charge (this boundary condition corresponds physically to requiring that the surface gravity, and hence the temperature, is constant along the horizon). This allows us to map the problem of finding a charged solution of the form (11) to finding a solution in the uncharged case.

One of the equations of motion can be solved algebraically for $A$ in terms of $K$; this leaves a system of three second-order equations which need to be satisfied by the function $K(R, v)$. Generically, such a system is heavily over-determined; however, it was shown in 19] that the system is consistent to second order in perturbation theory. This surprising result was elucidated in [20, 21], where it was shown that in the neutral case, the seemingly restricted ansatz taken above is in fact equivalent to the most general ansatz consistent with the symmetries. For the neutral black string, the Harmark and Obers ansatz reduces to

$$
d s_{n}^{2}=-f d t^{2}+R_{T}^{2}\left(f^{-1} A d R^{2}+\frac{A}{K^{d-2}} d v^{2}+K R^{2} d \Omega_{d-2}^{2}\right)
$$

where

$$
f=1-\frac{R_{0}^{d-3}}{R^{d-3}}
$$

Using staticity and spherical symmetry, the most general metric for a black string can be brought to the form

$$
d s^{2}=-e^{2 B} d t^{2}+e^{2 C}\left(d r^{2}+d z^{2}\right)+e^{2 D} d \Omega_{d-2}^{2}
$$

\footnotetext{
${ }^{4}$ We take the $v$ and $x^{i}$ coordinates to be periodically identified to allow us to write finite expressions.
} 
referred to as the conformal form, as the $(r, z)$ space is written in conformally flat coordinates. Here $B, C$ and $D$ are functions of $r$ and $z$ only. Since the latter form for the metric involves three arbitrary functions, while the former only involves two, it seems like the former must be more restrictive. However, they are in fact equivalent if we further assume the equations of motion are satisfied [20, 21]. To get from (11) to (9), we need to choose $R(r, z)$ so that $e^{2 B}=f$ is only a function of $R$, and $v(r, z)$ so that there is no $d R d v$ cross term in the resulting metric, and we get the appropriate relation between the $g_{R R}, g_{v v}$ and sphere components of the metric. These conditions can in fact be satisfied, subject to an integrability condition; in terms of the conformal form of the metric, this condition is

$$
\left(\partial_{r}^{2}+\partial_{z}^{2}\right) B+\left(\partial_{r} B\right)^{2}+\left(\partial_{z} B\right)^{2}+(d-2)\left(\partial_{r} B \partial_{r} D+\partial_{z} B \partial_{z} D\right)=0
$$

This condition is exactly the $R_{t t}=0$ equation of motion for the three-function conformal metric (11), so if the equations of motion are satisfied, we can pass from (11) to (9) by a coordinate transformation. In appendix A we discuss the extent to which this argument can be generalised to the charged case.

The important point for our present purpose is that this implies that any solution of the equations of motion describing a neutral black string, uniform or non-uniform, can be written in the form (9). This provides a convenient framework for discussing solutions. In 23], Harmark and Obers constructed a phase diagram summarising the known solutions in terms of two parameters, the mass $M$ and a relative binding energy $n$ (which provides a measure of the non-uniformity of the solutions). For general charge, the mass is

$$
M=\omega\left(R_{T} R_{0}\right)^{d-3}\left[(d-2)+(d-3) \sinh ^{2} \alpha\right]
$$

and the binding energy parameter is

$$
n=\frac{1-(d-2)(d-3) \chi}{(d-3) \sinh ^{2} \alpha+(d-2)-(d-3) \chi},
$$

where

$$
\omega=\frac{\Omega_{d-2} 2 \pi R_{T}}{16 \pi G_{N}},
$$

and $\chi$ parametrises the asymptotic falloff of the unknown function $K[19]$, and is hence independent of the charge.

The phase diagram for the neutral case given in [23] for a five-dimensional system on a circle of radius $R_{T}$ is sketched in figure 1

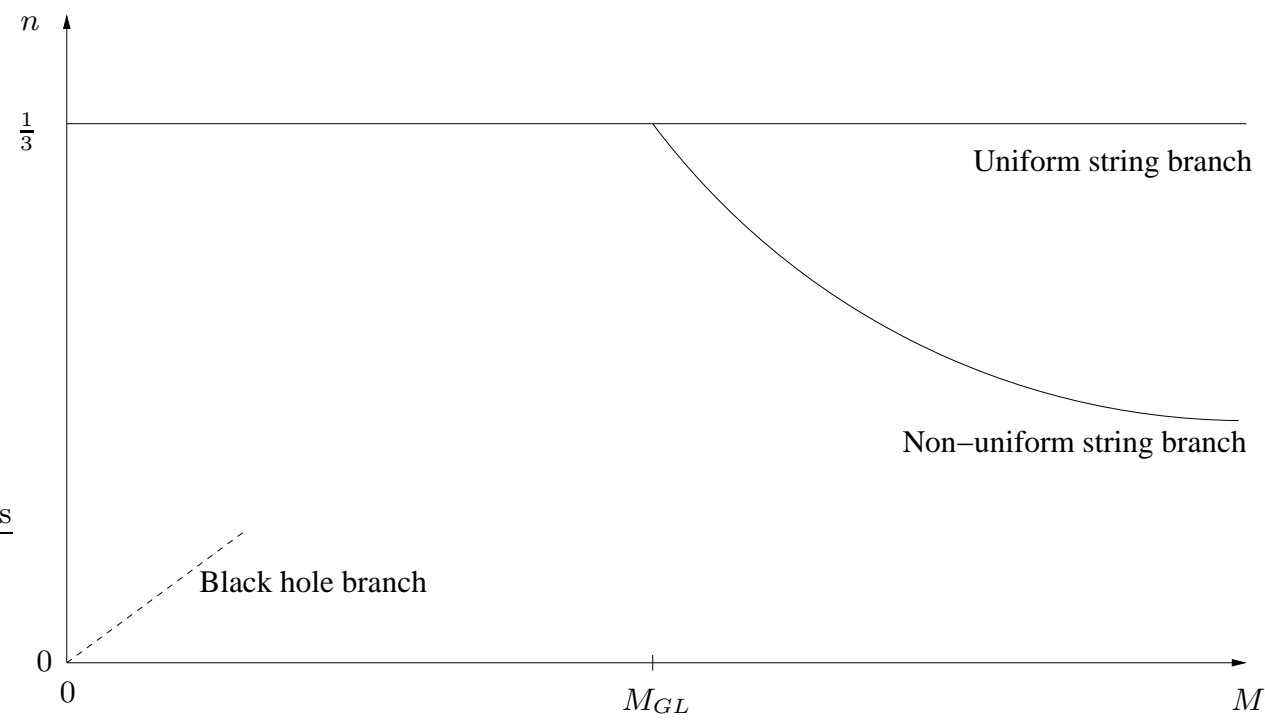

FIG. 1: Phase diagram for neutral solutions for a five-dimensional system on a circle.

Now since the equations of motion are independent of the charge, each solution in figure 1 gives a solution for every value of the charge. Inspection of (1314) shows that adding charge increases the mass, as expected, and decreases $n$, enhancing the binding effect. Thus, if we plot $M$ vs $n$ in the charged case, we get a qualitatively similar picture, as shown in figure 2 


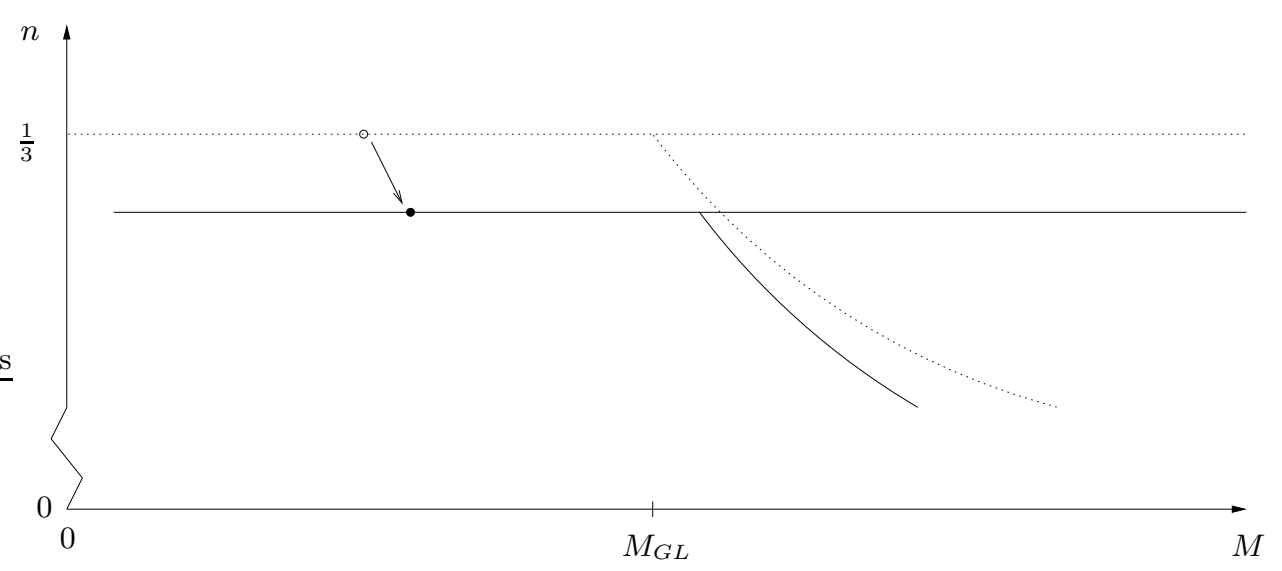

FIG. 2: The bold lines now refer to charged solutions. The diagram is qualitatively the same as in the neutral situation, shown as dotted lines for reference.

We can see that on a circle of some fixed radius $R_{T}$, there is always some critical value of the mass at which a non-uniform branch joins on to the uniform smeared black hole branch. We can re-state this in terms relevant for the Gubser-Mitra conjecture: for any given value of the mass and charge, there is a finite wavelength at which a threshold unstable mode occurs. We know that for zero charge, the uniform black string is unstable to modes of longer wavelength. Although we have not demonstrated the existence of the corresponding dynamical instability explicitly in the charged case, the persistence of the threshold unstable mode is strong evidence that it exists.

This result should be contrasted with the analysis of $p$-brane solutions in [8, 9, 10]. In those studies, it was found that for the ten-dimensional supergravity $p$-brane solutions with $p \leq 4$, there is a threshold unstable mode for the neutral case, but this mode goes off to infinite wavelength at a critical value of the charge, signalling the disappearance of the instability. This was found to occur at the same critical value of the charge where the specific heat changes sign.

In our case, by contrast, the threshold unstable mode exists all the way up to extremality, even though the specific heat changes sign before we reach extremality for cases with $d>5$. Thus, there are smeared branes which are locally thermodynamically stable, but possess a dynamical instability by the argument of the previous section. This is a clear violation of the Gubser-Mitra conjecture. Note that the wavelength of the threshold unstable mode, which signals the onset of instability, is determined by $R_{0}$, since the equations for $K$ are independent of charge. Hence if we go near extremality by taking $R_{0} \rightarrow 0$ and $\alpha \rightarrow \infty$ keeping $M$ fixed, the wavelength of the unstable mode will go to zero, suggesting that the instability will appear sufficiently close to extremality for any compactified black string as well.

\section{Acknowledgments}

We thank Ruth Gregory for discussions and collaboration at an early stage of this project. We would also like to thank Troels Harmark, Neils Obers and Mukund Rangamani for useful discussions. PB was supported by a PPARC studentship. SFR was supported by the EPSRC.

\section{APPENDIX A: GENERALITY IN THE CHARGED CASE}

In section III we reviewed the argument of 20, 21] that the Harmark and Obers ansatz is consistent in the neutral case. Since the equations for the unknown functions $A(R, v)$ and $K(R, v)$ are independent of the charge, this also implies that the ansatz is consistent in the charged case. It would still be interesting, however, to ask if we can show that the most general solution of the equations of motion with the assumed symmetries can be written in the form (12) when we include charge.

We can easily show that the metric can be written in the form (1) by an extension of the previous argument. 
Starting from the 3-function conformal form (11), we can make the redefinitions,

$$
\begin{aligned}
& e^{B} \rightarrow \bar{H}^{-\frac{d-2}{n-2}} e^{B}, \\
& e^{C} \rightarrow \bar{H}^{\frac{1}{n-2}} e^{B} \\
& e^{D} \rightarrow \bar{H}^{\frac{1}{n-2}} e^{D}
\end{aligned}
$$

for any function $\bar{H}$, so that (11) becomes

$$
d s^{2}=\bar{H}^{-\frac{d-2}{n-2}}\left(-e^{2 B} d t^{2}+\bar{H} e^{2 C}\left(d r^{2}+d z^{2}\right)+\bar{H} e^{2 D} d \Omega_{d-2}^{2}\right) .
$$

If we now perform the same change of variables as was used in 21] in the neutral case,

$$
\begin{aligned}
R^{d-3} & =R_{0}^{d-3}+\bar{r}^{d-3} \\
\hat{A} & =f^{-1} A R_{T}^{2}\left(\frac{\bar{r}}{R}\right)^{2(d-4)} \\
\hat{K}^{d-2} & =\frac{K^{d-2}}{f}\left(\frac{\bar{r}}{R}\right)^{2(d-4)}
\end{aligned}
$$

and then transform to the conformal form by making the further transformation,

$$
\begin{array}{cl}
\bar{r}=g(r, z), & v=h(r, z) \\
\partial_{r} g=e^{-(d-2) k} \partial_{z} h, \quad & \partial_{z} g=e^{-(d-2) k} \partial_{r} h .
\end{array}
$$

We can bring the metric (1) in the ansatz to the form (A4) if

$$
g^{d-3}=\frac{R_{0}^{d-3} e^{2 B}}{1-e^{2 B}}
$$

and

$$
e^{2 a}=\frac{e^{2 c}}{\left(\partial_{r} g\right)^{2}+\left(\partial_{z} g\right)^{2}}, \quad e^{2 k}=\frac{R_{T}^{2}}{R_{0}^{2}} e^{2 D} e^{\frac{2(d-5)}{(d-2)(d-3)} B}\left(1-e^{2 B}\right)^{\frac{2}{d-3}} .
$$

The system of equations in (A9) imply an integrability condition which together with (A11) imply that

$$
\left(\partial_{r}^{2}+\partial_{z}^{2}\right) B+\left(\partial_{r} B\right)^{2}+\left(\partial_{z} B\right)^{2}+(d-2)\left(\partial_{r} B \partial_{r} D+\partial_{z} B \partial_{z} D\right)=0,
$$

the same integrability condition we had in the neutral case.

If we assume that the arbitrary function $\bar{H}$ introduced in the redefinitions A1 identified with the dilaton as in (2), i.e., $e^{a \phi}=\bar{H}^{2}$, we can show that this integrability condition is again implied by the equations of motion. The most general form for $F_{\mu \nu}$ consistent with the assumed symmetries has only $F_{t z}$ and $F_{t r}$ non-zero; hence we can write

$$
F_{\lambda t} F_{t}^{\lambda}=\frac{1}{2} g_{t t} F^{2}
$$

and so eliminating the dilation field using (8) from the $t, t$ component of the graviton equation (7) gives us

$$
R_{t t}=g_{t t} \frac{n-3}{2(n-2)} \nabla^{2}(\ln \bar{H}) .
$$

This equation reduces to exactly (A12) for the metric given in equation (A4) and therefore we conclude that this integrability condition is implied by the equations of motion.

However, this is not yet enough to show that the general solution takes the form (12): we have not yet shown that $\bar{H}=H(R)$, and we have no coordinate freedom left to redefine it. The problem can be simply stated in coordinateindependent terms: in the charged case, there are two a priori independent scalar quantities, namely the norm of the timelike Killing vector $\partial_{t}$ and the dilaton. The ansatz (12) assumes a specific functional form for both of these. While we can choose coordinates so that one of them takes the specified form, it will not be possible to do this for both of them in general, without using some additional information. 
Thus, while it seems quite natural to us to assume that the ansatz (12) describes the most general solution of the equations of motion in the charged case as well, we cannot show this by some analogue of the arguments in 20, 21]. Rather, verifying our belief would require explicitly solving the equations of motion. We reiterate that this question of generality is irrelevant to the argument in the body of the paper, which required only the observation that uncharged solutions of the form (12) lift to charged solutions.

[1] J. M. Bardeen, B. Carter and S. W. Hawking, "The Four Laws Of Black Hole Mechanics," Commun. Math. Phys. 31 (1973) 161.

[2] J. D. Bekenstein, "Black Holes And Entropy," Phys. Rev. D 7 (1973) 2333.

[3] R. Gregory and R. Laflamme, "Black Strings And P-Branes Are Unstable," Phys. Rev. Lett. 70,2837 (1993) arXiv:hep-th/9301052.

[4] R. Gregory and R. Laflamme, "The Instability of charged black strings and p-branes," Nucl. Phys. B428, 399 (1994) arXiv:hep-th/9404071.

[5] S. S. Gubser and I. Mitra, "Instability of charged black holes in anti-de Sitter space," arXiv:hep-th/0009126 ibid, "The evolution of unstable black holes in anti-de Sitter space," JHEP 0108 (2001) 018 arXiv:hep-th/0011127.

[6] H. S. Reall, "Classical and Thermodynamic Stability of Black Branes," Phys. Rev. D64 (2001) 044005 arXiv:hep-th/0104071.

[7] J. P. Gregory and S. F. Ross, "Stability and the negative mode for Schwarzschild in a finite cavity," Phys. Rev. D 64 (2001) 124006 arXiv:hep-th/0106220.

[8] T. Hirayama, G. W. Kang and Y. O. Lee, "Classical stability of charged black branes and the Gubser-Mitra conjecture," Phys. Rev. D 67 (2003) 024007 arXiv:hep-th/0209181.

[9] S. S. Gubser and A. Ozakin, "Universality classes for horizon instabilities," JHEP 0305 (2003) 010 arXiv:hep-th/0301002.

[10] G. Kang and J. Lee, "Classical stability of black D3-branes," JHEP 0403 (2004) 039 arXiv:hep-th/0401225.

[11] T. Hirayama and G. Kang, "Stable black strings in anti-de Sitter space," Phys. Rev. D 64 (2001) 064010 arXiv:hep-th/0104213.

[12] V. E. Hubeny and M. Rangamani, "Unstable horizons," JHEP 0205 (2002) 027 arXiv:hep-th/0202189.

[13] S. A. Hartnoll, "Instability of generalised AdS black holes and thermal field theory," JHEP 0308 (2003) 019 arXiv:hep-th/0305001; ibid, "Casimir effect and thermodynamics of horizon instabilities," arXiv:hep-th/0403281

[14] G. T. Horowitz and K. Maeda, "Fate of the black string instability," Phys. Rev. Lett 87 (2001) 131301 arXiv:hep-th/0105111.

[15] S. S. Gubser, "On non uniform black branes," Class. Quant. Grav. 19 4825-4844 (2002) arXiv:hep-th/0110193.

[16] T. Wiseman, "Static axisymmetric vacuum solutions and non-uniform black strings," Class. Quant. Grav. 20 (2003) 1137-1176 arXiv:hep-th/0209051.

[17] E. Sorkin, "A critical dimension in the black-string phase transition," arXiv:hep-th/0402216

[18] M. W. Choptuik, L. Lehner, I. Olabarrieta, R. Petryk, F. Pretorius and H. Villegas, "Towards the final fate of an unstable black string," Phys. Rev. D 68 (2003) 044001 arXiv:gr-qc/0304085.

[19] T. Harmark and N. A. Obers, "Black holes on cylinders," JHEP 05 (2002) 032 arXiv:hep-th/0204047.

[20] T. Wiseman, "From black strings to black holes," Class. Quant. Grav. 20 (2003) 1177 arXiv:hep-th/0211028.

[21] T. Harmark and N. A. Obers, "Phase Structure of Black Holes and Strings on Cylinders," Nucl. Phys. B 684 (2004) 183 arXiv:hep-th/0309230.

[22] D. Marolf and B. C. Palmer, "Gyrating strings: A new instability of black strings?," arXiv:hep-th/0404139

[23] T. Harmark and N. A. Obers, "New phase diagram for black holes and strings on cylinders," Class. Quant. Grav. 21 (2004) 1709 arXiv:hep-th/0309116. 